\title{
Phylogenetic Evidence for the Transfer of Eubacterium suis to the Genus Actinomyces as Actinomyces suis comb. nov.
}

\author{
W. LUDWIG, ${ }^{1 *}$ G. KIRCHHOF, ${ }^{1}$ M. WEIZENEGGER, ${ }^{1}$ AND N. WEISS ${ }^{2}$ \\ Lehrstuhl für Mikrobiologie, Technische Universität, D-8000 Munich, ${ }^{1}$ and Deutsche Sammlung von \\ Mikroorganismen und Zellkulturen, D-3300 Braunschweig, ${ }^{2}$ Germany
}

\begin{abstract}
The 16 S rRNA primary structures of Eubacterium suis DSM $20639^{\mathrm{T}}$ ( $\mathrm{T}=$ type strain) and Bifidobacterium bifidum DSM $20456^{\mathrm{T}}$ were determined by sequencing in vitro amplified rDNA. Sequence comparisons indicated that $B$. bifidum is moderately related to representatives of the genera Actinomyces and Mobiluncus. The closest relative of $E$. suis is Actinomyces pyogenes. $E$. suis and $A$. pyogenes are more closely related phylogenetically to one another than to the other Actinomyces species that have been investigated by using comparative $16 \mathrm{~S}$ rRNA analysis. Therefore, we propose that $E$. suis should be transferred to the genus Actinomyces as Actinomyces suis comb. nov.
\end{abstract}

Eubacterium suis Wegienek and Reddy 1982, a commonly occurring swine pathogen (23), was originally isolated by Soltys and Spratling in 1957 (20). The name "Corynebacterium suis" was proposed for this bacterium because of the diphtheroid morphology of the anaerobic organism. In 1982 in a taxonomic study, Wegienek and Reddy described the morphological, cultural, and biochemical characteristics of this organism and proposed the name Eubacterium suis. Corynebacteria are aerobic to facultatively anaerobic, contain arabinose, galactose, and meso-diaminopimelic acid as the major cell wall components, and produce major amounts of propionate in carbohydrate metabolism; in contrast, $\mathbf{E u}$ bacterium suis is anaerobic and contains rhamnose and lysine as the major cell wall components, and propionate is not among the end products of carbohydrate metabolism. Despite differences in cell wall composition and DNA base ratios ( $55 \mathrm{~mol} \% \mathrm{G}+\mathrm{C}$ for Eubacterium suis versus 31 to 45 mol\% $\mathrm{G}+\mathrm{C}$ for other members of the genus Eubacterium) $(11,13)$ and on the basis of a rather limited number of common characteristics, such as anaerobiosis, morphology, similar cultural conditions, and the absence of propionate, lactate, and succinate among the end products of carbohydrate metabolism, Wegienek and Reddy proposed that the organism should be included in the genus Eubacterium.

The genus Eubacterium is mainly defined by negative characteristics and represents a heterogeneous collection of gram-positive, nonsporeforming, obligately anaerobic rods $(11,13)$. In particular, the wide range of genomic DNA base ratios indicates that the genus includes organisms that are not related phylogenetically.

At present, comparative sequence analysis of rRNA is one of the most powerful methods for determining higher phylogenetic relationships among bacteria. In order to elucidate the phylogenetic affiliations of Eubacterium suis, we sequenced the greater part of its $16 \mathrm{~S}$ genes coding for rRNA (rDNA). The $16 \mathrm{~S}$ rDNA of Bifidobacterium bifidum was analyzed as a reference rDNA in addition to the previously published sequences for gram-positive bacteria with high DNA $\mathrm{G}+\mathrm{C}$ contents.

\footnotetext{
${ }^{*}$ Corresponding author.
}

\section{MATERIALS AND METHODS}

Bacterial strains and culture conditions. $B$. bifidum DSM $20456^{\mathrm{T}}\left(\mathrm{T}=\right.$ type strain) was cultured anaerobically at $37^{\circ} \mathrm{C}$ in a medium which contained (per liter of distilled water) 10 $\mathrm{g}$ of tryptone, $5 \mathrm{~g}$ of meat extract, $5 \mathrm{~g}$ of yeast extract, $10 \mathrm{~g}$ of glucose, $3 \mathrm{~g}$ of $\mathrm{K}_{2} \mathrm{HPO}_{4}, 5 \mathrm{~g}$ of $\mathrm{NaCl}, 10 \mathrm{ml}$ of Tween 80 , $10 \mathrm{~g}$ of sodium ascorbate, and $0.5 \mathrm{~g}$ of cysteine hydrochloride (pH 6.8). Eubacterium suis DSM $20639^{\mathrm{T}}$ was grown anaerobically at $37^{\circ} \mathrm{C}$ in peptone yeast extract maltose medium; the peptone yeast extract glucose medium described in the 1990 Catalog of strains of the Deutsche Sammlung von Mikroorganismen und Zellkulturen (6a) was modified by replacing glucose with $5 \mathrm{~g}$ of maltose per liter.

Cell wall analysis. The analysis of cell wall components was performed as described by Schleifer and Kandler (19).

Sequence analysis. Genomic DNA was purified by using the Marmur method (12). 16S rDNA was amplified in vitro by using the polymerase chain reaction technique (14) in combination with oligonucleotide primers identical or complementary to highly conserved regions of the rRNAs. The amplified rDNA fragments were cloned in the vector pBluescript (Stratagene, LaJolla, Calif.) and Escherichia coli JM83. The cloned rDNA and the amplified rDNA were sequenced by using the chain termination method $(4,16)$ and site-specific oligonucleotide primers. The oligonucleotides

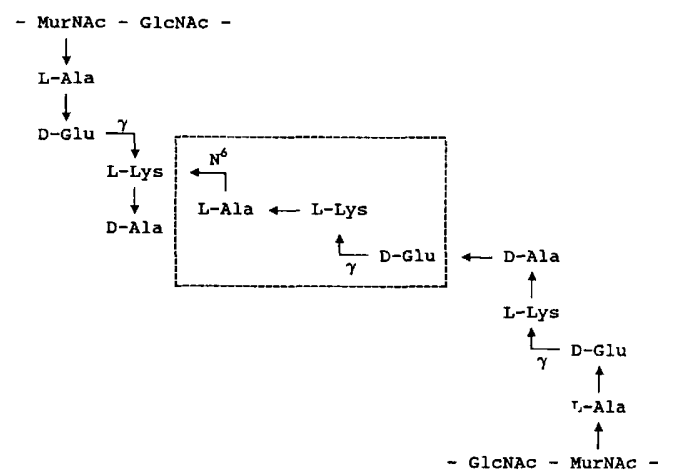

FIG. 1. Fragment of the peptidoglycan primary structure of Eubacterium suis. Abbreviations: Ala, alanine; GlcNAc, $N$-acetylglucosamine; Glu, glutamic acid; Lys, lysine; MurNAc, $N$-acetylmuramic acid. 
1

Es nnnmmagegtttgatnntggctcagnnmmmnnnnCGGCGTGCTTAACACATGCAAGTCGAACGGAMGGCTTCTTTCTTITTTGAT

AP IITGTGGAGAGTIMATCCTGGCTCAGGACGAACGCTGGCGGCGTGCTTAACACATGCAGTCGAACGATGAAGCCGGGn-CnnTT --GTT

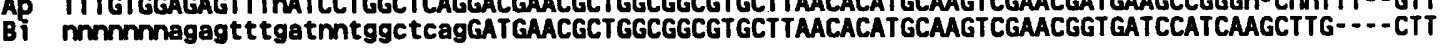

93

184

Es GGAGTGCTCGAGTGGCGAACGGGTGAGTAATACGTGGGTAACCTGCCCTCAACTGTGGGATAAGCTTGGGAAACTGGGTCTAATACTGCATA

AP TTGGTGGATTAGTGGCGACGGGTGAGTMAMCGTGAGTAACCTGCCCTTGTCTITGGGATAAGCCTGGGAAACTGGGTCTAATACCGGATA

Bì GGTGGTGATGAGTGGCAMCGGGTGAGTAATGCGTGACCGACCTGCCCCAGACTCCGGAATAGCTCCTGGAAACGGGTGGTAATGCCGGATG

185

276

Es IICTTCTGTCCTCGCATGGGGGTGGTTGGAAAG-TTTITICGGTTGGGGATGGGCTCGCGGCCTATCAGTITGTTGGTGGGGTGATGGCCTA

AP TTCTGCTTITGCCGCATGGTGGGGGTTGGMAGATTTTTT-GGATGGGNIGGNCTCACGGCCTATCAGCTTGTTGGTGGGGTGATGGCCTA

Bi TTCCAC-ATGATCGCATGTGATT-GTGGGAAAGATTCTATCGGCGTGGGATGGGGTCGCGTCCTATCAGCTTGTTGGTGAGGTAACGGCTCA

277

368

Es CCMGGGGTTGACGGGTAGCCGGCCTGAGAGGGTGGTCGGCCACATTGGGACTGAGATACGGCCCAGACTCCTACGGGAGGCAGCAGTGGGG

AP CCAAGGCGTCGA CGGGTAGCCGGCCTGAGAGGGT GACCGGCCACATTGGGACTGAGATACGGCCINGACTCCTACGGGAGGCAGCAGTGGGG

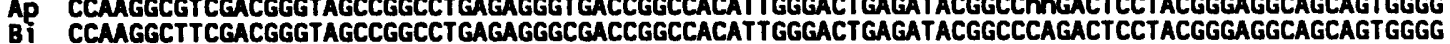

369

460

Es GATATTGCACAATGGGCGGAAGCTTGATGCAGCGACGCCGCGTGGGGGATGAAGGCITTCGGGITGTAAACTICTIITIGGTGCAGAACAAGG

AD AMTATTGCACAMTGGACGCAAGTCTGATGCAGCGACGCCGCGTGGGGGATGAAGGCTTICGGGTTGTAAACTCCTTTCAGTACAGAAGAAGC

Bi AATATTGCACMTGGGCGCAGCCTGATGCAGCGACGCCGCGTGAGGGATGGAGGCCTTCGGGTTGTAAACCTCTTITGTTIGGGAGCAAGC

461

552

Es CTCGCACTTGGGGTGGGITGGGGTATGTGCTGAATMGGGCCGGCTMACTACGTGCCAGCAGCCGCGGTAATACGTAGGGCGCGAGCGTTG

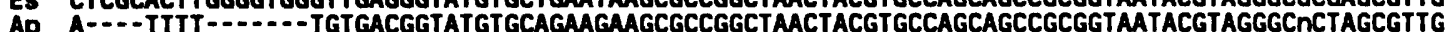

Bi C-.--TICG-...--GGTGAGTGTACCTTTCGATAMGCGCCGGCTAACTACGTGCCAGCAGCCGCGGTAATACGTAGGGCGCAAGCGTTA

553

644

Es TCCGGAATTATTGGGCGTAAAGGGCTTGTAGGCGGCTTGTTGCGCCTGCTGTGAAAACGCGGGGCTTAACTTCGCGCGTGCAGTGGGTACGG

AD TCCGGMATTATTGGGCGTAAGAGCTCGTAGGCGGTTTGTTGCGCCTGCTGTGAAAGACCGGGGCTTMACT TCGGNGTTGCAGTGGGTACGG

Bi TCCGGATTTATTGGGCGTAAAGGGCTCGTAGGCGGCTCGTCGCGTCCGGTGTGAAAGTCCATCGCTIAACGGTGGATCTGCGCCGGGIACGG

645

736

Es GCAGGCTTGAGTGTGGTAGGGGTGACTGGAATTCCAGGTGTAGCGGTGGAATGCGCAGATATCTGGAGGAATACCGATGGCGAAGGCAGGTC

Ap GCAGACTAGAGTGTGGTAGmm GATTGGnATICCTGGTGTAGCGGTGGATGCGCAGATATCAGGAGGACACCGATGGCGAAGGnnGn

Bi GCGGGCTGGAGTGCGGTAGGGGAGACTGGAATTCCCGGTGTAACGGTGGAATGTGTAGATATCGGGAAGAACACCGAT GGCGAAGGAGGTC

$737 \quad 828$

Es ACTGGGCCATTACTGACGCTGAGGAGCGAAGCGTGGGTAGCGACAGGATTAGATACCCTGGTAGTCCATGCTGTAAACGTTGGGAACTAG

AP ACTGGGCCATTACTGACGCTGAGGAGCGAAAGCGTGGGTAGCGAACAGGATTAGATACCCTGGTAGTCCACGCTGTAAACGTTGGGCACTAG

Bi TCTGGGCCGTCACTGACGCTGAGGAGCGAAGCGTGGGGAGCGAACAGGATTAGATACCCTGGTAGTCCACGCCGTAAACGGTGGACGCTGG 829

920

Es GTGTGGGGTCCTITTCCGGGGTTCTGCGCCGTAGCTAACGCTTTAAGITCCCCGCCTGGGGAGTACGGCCGCAAGGCTAAACTCAMGGAA

Ap GTGTGGGGCCTITICCACGGGTTCTGCGCCGTAnnTAACGCTTTAAGTGCCCCGCCTGGGGAGTACGGCCGCAGGGTIAAACTCAAAGGA

Bi ATGTGGGGCACGTTCCACGTGTTCCGTGTCGGAGCTAACGCGTTAAGCGTCCCGCCTGGGGAGTACGGCCGCAAGGCTAAAACTCAAAGAAA

921

1012

Es TrGACGGGGGCCCGCACAAGCGGCGGAGCATGCGGATTAATTCGATGCAACGCGAAGAACCTTACCAAGGCTTGACATACATTGCGATATIC

Ap TTGACGGGGGCCCGCACAAGCGGCGGAGCATGCGGATTAATTCGATGCAACGCGAGGAACCTTACCAAGGCTTGACATACACTGCGATGTGC

Bi TTGACGGGGGCCGCACAAGCGGCGGAGCATGCGGATTAATTCGATGCAACGCGAAGAACCTTACCTGGGCTTGACATGITCCCGACGACGC

1013

Es CAGAGATGGGGTAGCT-TTTTT-GGTGGTGTACAGGTGGTGCATGGTTGTCGTCAGCTCGTGTCGTGAGATGTTGGGTTAAGTCCCGCAACG

AP CAGAGATGGTGCAGCC-TICGG-GGTGGTGTACAGGTGGIGCATGGTTGTCGTCAGCTCGTGTCGTGAGATGITGGGTIAAGTCCCGCAACG

Bi CAGAGATGGCGTTTCCCTTCGGGCGGGTTCACAGGTGGTGCATGGTCGTCGTCAGCTCGTGTCGTGAGATGTTGGGTTAAGTCCCGCAACG 1105

1196

Es AGCGCAACCCTTGTCTTGTGTTGCCAGCGGTTTTGGCCGGGGACTCACGGGAGACTGCCGGGGTTAACTCGGAGGAAGGTGGGGATGACGTC

Ap AGCGCAACCCTTGTCCTGTGTTGCCAGCATGTTGTGGTGGGGACTCACGGGAGACTGCCGGGGTIAACTCGNAGGAAGGTGGGGATGACGTC

Bi î AGCGCAACCCTCGCCCCGTGTTGCAGCACGTTATGGTGGGAACTCACGGGGACCGCCGGGGTIAACTCGGAGGAGGGGGGGATGACGTC 1197

1288

Es AAATCATCATGCCCCTIATGTCTTGGGCTTCACGCATGCTACAATGGCCGGTACAGTGGGTTGCGATACTGTGAGGTGGAGCGAATCTCTGA

Ap AMATCATCATGCCCNTIATGTCTTGGGCTTCACGCATGCTACAATGGCCGGTACAGAGGGTTGCGAGCCTGTGAGGGTGAGCTAATCCCTTA

Bì AGATCATCATGCCCCTIACGTCCAGGGCTTCACGCATGCTACAATGGCCGGTACAGCGGGATGCGACATGGCGACATGGAGCGGATCCCTGA 1289

1380

Es AAGCCGGTCTCAGTTCGGATCGGGGTCTGCAACTCGACCTCGTGAAGTGGGAGTCGCTAGTAATCGCGGATCAGCATTGCCGCGGTGAATAC

Ap AAGCTnGTCTCAGTTCGGATTGGGGTCTGCAACTCGACCCCATGNAGnCGGAGTCGCTAGTAATCGCAGATCAGCAACGCTGCGGTGATAC 1381

Es GTTCCCGGGCCTTGTACACACCGCCCGTCACGTCACGAAAGTTGGCGACACCCGAAGCTCGTGGCCTAACCA-TTTTTGGGGGGAGCGGTCG

AP GTmmmnnmm

Bi GTTCCCGGGCCTTGTACACACCGCCCGTCAAGTCATGAAAGTGGGCAGCACCCGAAGCCGGTGGCCTAACCCCTTGTGGGATGGAGCCGTCT

1473

Es MAGGTGGGGTTGGCGATTGGGACGAaGTCGTAACAAGCTAGCCGTACCGGAAGGTGCGGCTGGATCACCTCCTTTCT

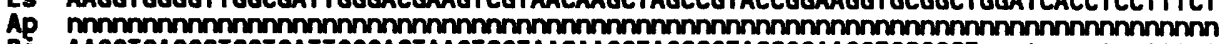

Bi MGGTGAGGCTCGTGATTGGGACTAGTCGTAACAAGGTAGCCGTACCGGAAGGTGCGCTggatcace tect tet

FIG. 2. Alignment of the $16 \mathrm{~S}$ rRNA primary structures of Eubacterium suis (Es), B. bifidum (Bb), and A. pyogenes (Ap) (22). Lower-case letters indicate target sites of amplification primers; $n$ indicates a base that was not determined or was not unambiguously determined. 
TABLE 1 . Similarity matrix ${ }^{a}$

\begin{tabular}{|c|c|c|c|c|c|c|c|c|c|c|c|c|c|}
\hline \multirow[b]{2}{*}{ Organism } & \multirow[b]{2}{*}{$\begin{array}{l}\text { No. of } \\
\text { bases }^{b}\end{array}$} & \multicolumn{12}{|c|}{$\%$ of similarity with: } \\
\hline & & 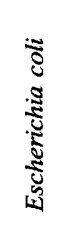 & 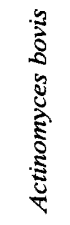 & 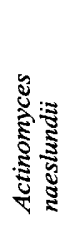 & 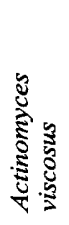 & 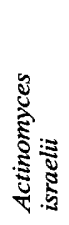 & 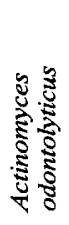 & 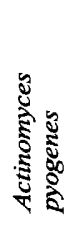 & 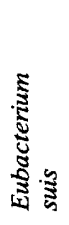 & 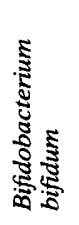 & 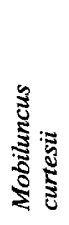 & 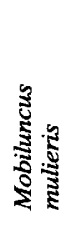 & 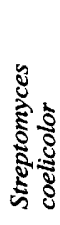 \\
\hline Escherichia coli & 1,542 & & 75.2 & 74.3 & 74.4 & 72.4 & 74.4 & 77.1 & 74.6 & 75.9 & 73.7 & 74.6 & 77.3 \\
\hline Actinomyces bovis & 1,341 & 80.1 & & 93.9 & 94.9 & 93.1 & 91.7 & 89.1 & 88.5 & 85.9 & 89.1 & 88.5 & 78.7 \\
\hline Actinomyces naeslundii & 1,346 & 79.8 & 97.3 & & 97.0 & 93.2 & 90.6 & 88.7 & 87.1 & 85.4 & 87.4 & 87.9 & 77.9 \\
\hline Actinomyces viscosus & 1,403 & 79.8 & 97.3 & 98.5 & & 94.2 & 91.7 & 89.3 & 88.0 & 85.3 & 88.4 & 88.0 & 78.6 \\
\hline Actinomyces israelii & 1,066 & 81.2 & 96.5 & 96.7 & 96.7 & & 89.7 & 88.4 & 87.3 & 84.8 & 88.1 & 88.9 & 77.0 \\
\hline Actinomyces odontolyticus & 1,317 & 79.2 & 95.1 & 95.1 & 95.3 & 93.6 & & 90.3 & 88.8 & 86.2 & 88.9 & 88.3 & 77.5 \\
\hline Actinomyces pyogenes & 1,326 & 81.2 & 92.5 & 92.5 & 93.1 & 91.6 & 92.5 & & 91.7 & 84.2 & 88.9 & 88.3 & 77.4 \\
\hline Eubacterium suis & 1,491 & 80.5 & 90.9 & 90.5 & 91.6 & 91.3 & 90.9 & 93.8 & & 83.6 & 88.1 & 87.5 & 76.0 \\
\hline Bifidobacterium bifidum & 1,523 & 79.8 & 87.8 & 88.5 & 88.7 & 88.2 & 87.4 & 86.7 & 87.1 & & 82.0 & 81.3 & 76.0 \\
\hline Mobiluncus curtesii & 1,318 & 79.2 & 92.0 & 91.6 & 92.2 & 91.4 & 92.0 & 93.4 & 92.5 & 84.5 & & 97.1 & 77.2 \\
\hline Mobilunus mulieris & 863 & 78.9 & 91.8 & 91.6 & 92.2 & 91.3 & 91.6 & 93.3 & 92.9 & 94.5 & 98.0 & & 77.8 \\
\hline Streptomyces coelicolor & 1,542 & 83.2 & 84.3 & 84.3 & 84.5 & 83.4 & 82.9 & 83.8 & 82.9 & 81.8 & 83.1 & 82.7 & \\
\hline
\end{tabular}

${ }^{a}$ Levels of $16 \mathrm{~S}$ rRNA sequence similarity between Actinomyces species and some reference gram-positive bacteria. Data were obtained from the following sources: Actinomyces spp., references 21 and 22; Bifidobacterium and Eubacterium spp., this study; Escherichia coli, reference 2; Mobiluncus spp., reference 10; and Streptomyces coelicolor, reference 1 . The values on the upper right are overall similarity values; the values on the lower left are values that were based on a data set that was reduced to those positions which were invariant in at least $50 \%$ of the aligned sequences.

$b$ Number of bases that were unambiguously determined.

were synthesized by using a Cyclone DNA synthesizer (Milligen Biosearch, Eschborn, Germany).

Data analysis. The $16 \mathrm{~S}$ rRNA sequences were added to an alignment of about 300 homologous sequences. A matrix of $K_{\text {nuc }}$ values (9) was established for the sequences which we determined and a selection of related sequences. Distance matrix trees were reconstructed by using the method of Fitch and Margoliash (8) or by using the neighbor joining method described by Saitou and Nei (15), using the FITCH program of the PHYLIP program package of Felsenstein (7) and the NJTREE program of Jin and Ferguson, respectively. Alignment gaps and positions that were not determined were not taken into consideration when we calculated binary distance values. Parsimony and bootstraped parsimony analyses of the sequence data were performed by using the DNAPARS, DNAINVAR, and DNABOOT programs as implemented in the PHYLIP package.

\section{RESULTS}

We determined the structure of the peptidoglycan moiety of the cell wall of Eubacterium suis. A fragment of the primary structure of the peptidoglycan is shown in Fig. 1. An approximately 1.8-kb DNA fragment from Eubacterium suis containing the greater part of a 16S rRNA gene, the intergeneric spacer, and a small $5^{\prime}$ portion of a $23 S$ rRNA gene was amplified in vitro by using the polymerase chain reaction technique and a pair of rDNA-specific primers. The nucleotide sequences of the primers were 5'-CATGCAAGTC GARTC-3' and 5'-GGGTTYCCCCATTCGG-3'; the corresponding target sites were homologous to bases 54 to 68 of Escherichia coli 16S rRNA and bases 115 to 130 of Escherichia coli $23 \mathrm{~S}$ rRNA, respectively. The amplified fragment was cloned in the vector pBluescript (Stratagene) as pES611-318. In addition, an alternative primer pair (5'-AGA GTTTGATYMGGCTCAG-3' and 5'-AGAAAGGAGGTGA TCC $-3^{\prime}$ ) was used, and the amplified rDNA was sequenced directly. The same pair of primers was used to amplify a $16 \mathrm{~S}$
rDNA fragment of $B$. bifidum which was homologous to positions 8 to 1542 of Escherichia coli 16S rRNA. The amplified 16S rDNA was cloned in pBluescript as pBbil6. The aligned 16S rDNA sequences from Eubacterium suis, $B$. bifidum, and Actinomyces pyogenes (22) are shown in Fig. 2.

The sequences were compared with a data set containing $6016 \mathrm{~S}$ rRNA sequences from representatives of grampositive bacteria with high DNA $\mathrm{G}+\mathrm{C}$ contents. The $16 \mathrm{~S}$ rRNA sequence of Escherichia coli (2) was used as an outgroup reference. A matrix of the binary similarity values for the organisms investigated in this study, as well as a selection of reference organisms, is shown in Table 1. Table 1 shows the overall similarity values, as well as values that were calculated from a data set reduced to those positions which were invariant in at least $50 \%$ of the sequences. Since most of the previously published $16 \mathrm{~S}$ rRNA sequences that were used as references have not been determined completely, the numbers of bases which were sequenced are shown for the individual sequences in Table 1. Eubacterium suis and its closest relative, A. pyogenes, exhibited 16S rRNA similarity values of $91.7 \%$ (full data set) and $93.8 \%$ (reduced data set); these values are remarkably higher than the lowest values for the Actinomyces species (87.1 and $90.5 \%$, respectively).

A phylogenetic tree based on corrected distance values $\left(K_{\text {nuc }}\right)$ is shown in Fig. 3.

\section{DISCUSSION}

The phylogenetic unity of five species of the genus Actinomyces (Actinomyces bovis, Actinomyces israelii, Actinomyces naeslundii, Actinomyces odontolyticus, and $A$. pyogenes) has been demonstrated previously by the results of a comparative $16 \mathrm{~S}$ rRNA sequence analysis (21). A. pyogenes (22) was not included in this study. However, this organism is related to the Actinomyces species that have been investigated on the rRNA level so far, but when its rRNA is compared with other Actinomyces rRNAs, its 16S rRNA 


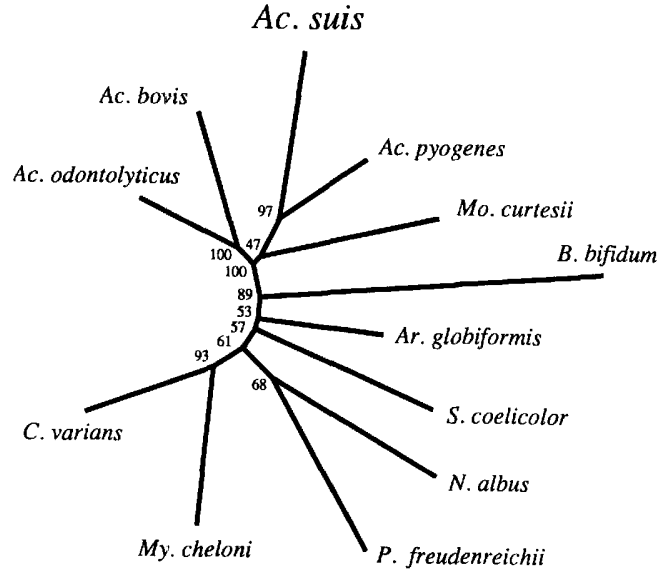

0.05 Knuc

FIG. 3. Phylogenetic tree showing the relationships among $E u$ bacterium suis, selected Actinomyces species, $B$. bifidum, and reference species of the major phylogenetic groups of gram-positive bacteria with high DNA $\mathrm{G}+\mathrm{C}$ contents. The tree was based on a matrix of distance values which was established by including only those alignment positions which were invariant in at least $50 \%$ of all sequences. The tree was reconstructed by using the neighbor joining method (15). The topology of the tree was reevaluated by performing a bootstrapped parsimony analysis. The numbers indicate the fractions of bootstrapping replicates in which the groups of related organisms separated at each node appeared to be monophyletic. Abbreviations (references): Ac., Actinomyces $(21,22) ;$ Ar., Arthrobacter $(25) ; B .$, Bifidobacterium (this study); C., Corynebacterium (6); Mo., Mobiluncus (10); My., Mycobacterium (4); N., Nocardioides (5); S., Streptomyces (1).

primary structure exhibits the lowest degree of sequence similarity.

The results of a $16 \mathrm{~S}$ rRNA analysis of Eubacterium suis show that this organism is a close relative of $A$. pyogenes. These two organisms share a common cell wall composition (lysine is the diamino acid; rhamnose and traces of mannose are the cell wall sugars), they both contain type $c$ cytochromes, and they have similar DNA G + C contents $(55$ and 56 to $58 \mathrm{~mol} \%$, respectively) $(17,18,20,23)$. Differences in the biochemical characteristics of Eubacterium suis Soltys and Spratling and the species incertae sedis "Actinomyces suis" Franke 1973 (13) indicate that these two organisms are not identical. Since no type strain of "A. suis" Franke has been validly described and the Eubacterium suis type strain is the validly described type strain of the species, we propose that Eubacterium suis should be transferred to the genus Actinomyces as Actinomyces suis comb. nov.

The affiliation of the genera Mobiluncus and Actinomyces has been shown by Lassnig et al. (10). An analysis of the now enlarged data set indicated that the phylogenetic relationships of the genera Mobiluncus and Actinomyces are at the same level as the lowest intragenus relationships among Actinomyces species. When different tree construction methods were used and the data set was varied by successively removing variable alignment positions, the position of the genus Mobiluncus was not stable in the tree. This is reflected by a low bootstrap confidence value for the $\mathrm{Mo}$ biluncus branch in Fig. 3. In most cases the genus Mobiluncus grouped with $A$. pyogenes and Eubacterium suis as shown in the tree in Fig. 3. At present, the position of the genus Mobiluncus cannot be determined unambiguously with respect to the species of the genus Actinomyces. Nevertheless, the genus Mobiluncus clearly can be separated from the genus Actinomyces by its different cell wall composition (24). B. bifidum, which was included in this study, is only moderately related to the genera Actinomyces and Mobiluncus, as postulated previously by Stackebrand and Charfreitag (21) on the basis of partial sequence data.

Description of Actinomyces suis (Wegienek and Reddy) comb. nov. Actinomyces suis (Eubacterium suis Wegienek and Reddy 1982; "Corynebacterium suis" Soltys and Spratling 1957). (su'is. L. n. sus, hog, swine; L. gen. n. suis, of a hog). The description of the species is the same as the description of Eubacterium suis given by Moore and Holdemann Moore (13). The type strain is strain DSM 20639 (= ATCC 33144)

\section{ACKNOWLEDGMENTS}

We thank I. Pomper and E. Waltenberger for technical assistance. This work was supported by a grant from the Bundesministerium für Forschung und Technologie and by the state of Lower Saxony.

\section{REFERENCES}

1. Baylis, H. A., and M. J. Bibb. 1987. The nucleotide sequence of a 16S rRNA gene from Streptomyces coelicolor A3(2). Nucleic Acids Res. 15:1776.

2. Brosius, J., J. L. Palmer, J. P. Kennedy, and H. F. Noller. 1978. Complete nucleotide sequence of a 16S ribosomal RNA gene from Escherichia coli. Proc. Natl. Acad. Sci. USA 75:48014805.

3. Charfreitag, O., M. D. Collins, and E. Stackebrandt. 1988 Reclassification of Arachnia propionica as Propionibacterium propionicum comb. nov. Int. J. Syst. Bacteriol. 38:345-357.

4. Chen, E. Y., and P. H. Seeburg. 1985. Supercoiled sequencing: a fast and simple method for sequencing plasmid DNA. DNA 4:165-170.

5. Collins, M. D., M. Dorsch, and E. Stackebrandt. 1989. Transfer of Pimelobacter tumescens to Terrabacter gen. nov. as Terrabacter tumescens comb. nov. and of Pimelobacter jensenii to Nocardioides as Nocardioides jensenii comb. nov. Int. J. Syst. Bacteriol. 39:1-6.

6. Collins, M. D., J. Smida, and E. Stackebrandt. 1989. Phylogenetic evidence for the transfer of Caseobacter polymorphus (Crombach) to the genus Corynebacterium. Int. J. Syst. Bacteriol. 39:7-9.

6a.Deutsche Sammlung von Mikroorganismen und Zellkulturen. 1990. Catalog of strains. Deutsche Sammlung von Mikroorganismen und Zellkulturen, Braunschweig, Germany.

7. Felsenstein, J. 1982. Numerical methods for inferring evolutionary trees. Q. Rev. Biol. 57:379-404.

8. Fitch, W. M., and E. Margoliash. 1967. Construction of phylogentic trees: a method based on mutation distances as estimated by cytochrome $c$ sequences is of general applicability. Science 155:279-284.

9. Hori, H., and S. Osawa. 1979. Evolutionary change in 5S rRNA secondary structure and phylogenetic tree of 54 5S rRNA species. Proc. Natl. Acad. Sci. USA 76:381-385.

9a.Jin and Ferguson. Personal communication.

10. Lassnig, C., M. Dorsch, J. Wolters, E. Schaber, G. Stöffler, and E. Stackebrandt. 1989. Phylogenetic evidence for the relationship between the genera Mobiluncus and Actinomyces. FEMS Microbiol. Lett. 65:17-22.

11. Lewis, R. P., and V. L. Sutter. 1981. The genus Eubacterium, p. 1903-1911. In M. P. Starr, H. Stolp, H. G. Trüper, A. Balows, and H. G. Schlegel (ed.), The prokaryotes. Springer Verlag, Berlin.

12. Marmur, J. 1961. A procedure for the isolation of DNA from microorganisms. J. Mol. Biol. 3:208-218.

13. Moore, W. E. C., and L. V. Holdemann Moore. 1986. Genus Eubacterium Prévot 1938, 294 ${ }^{\mathrm{AL}}$, p. 1353-1373. In P. H. A. 
Sneath, N. S. Mair, M. E. Sharpe, and J. G. Holt (ed.), Bergey's manual of systematic bacteriology, vol. 2. The Williams \& Wilkins Co., Baltimore.

14. Saiki, R. K., D. H. Gelfand, S. Stoffel, S. J. Scharf, R. Higuchi, G. T. Horn, K. G. Mullis, and E. H. Ehrlich. 1988. Primer directed enzymatic amplification of DNA with a thermostable DNA polymerase. Science 239:487-491.

15. Saitou, N., and M. Nei. 1987. The neighbour-joining method: a new method for reconstructing phylogenetic trees. Mol. Biol. Evol. 4:406-425.

16. Sanger, F., S. Nicklen, and A. R. Coulson. 1977. DNA sequencing with chain terminating inhibitors. Proc. Natl. Acad. Sci. USA 74:5463-5467.

17. Schaal, K. P. 1986. Genus Actinomyces Harz $1877,133^{\mathrm{AL}}$, p. 1383-1418. In P. H. A. Sneath, N. S. Mair, M. E. Sharpe, and J. G. Holt (ed.), Bergey's manual of systematic bacteriology, vol. 2. The Williams \& Wilkins Co., Baltimore.

18. Schaal, P., and G. Pulverer. 1981. The genera Actinomyces, Agromyces, Arachnia, Bacterionema, and Rothia, p. 19231950. In M. P. Starr, H. Stolp, H. G. Trüper, A. Balows, and
H. G. Schlegel (ed.), The prokaryotes. Springer Verlag, Berlin. 19. Schleifer, K. H., and O. Kandler. 1972. Peptidoglycan types of bacterial cell walls and their taxonomic implications. Bacteriol. Rev. 36:407-477.

20. Soltys, M. A., and F. R. Spratling. 1957. Infectious cystidis and pyelonephritis in pigs: a preliminary communication. Vet. Rec. 69:500-504.

21. Stackebrandt, E., and O. Charfreitag. 1990. Partial 16S rRNA primary structure of five Actinomyces species: phylogenetic implications and development of an Actinomyces israeliispecific oligonucleotide probe. J. Gen. Microbiol. 136:37-43.

22. Stahl, D. A., and J. W. Urbance. 1990. The division between fast- and slow-growing species corresponds to natural relationships among the mycobacteria. J. Bacteriol. 172:116-124.

23. Wegienek, J., and C. A. Reddy. 1982. Taxonomic study of "Corynebacterium suis"' Soltys and Spratling: proposal of Eubacterium suis (nom. rev.) comb. nov. Int J. Syst. Bacteriol. 23:218-228.

24. Weiss, N. Unpublished data

25. Woese, C. R. EMBL accession no. M23411. 